\title{
Targeting CDC7 improves sensitivity to chemotherapy of esophageal squamous cell carcinoma
}

This article was published in the following Dove Press journal: OncoTargets and Therapy

\author{
Ji-Xiang $\mathrm{Cao}^{1,2, *}$ \\ Yao $\mathrm{Lu}^{3, *}$
}

'Key Laboratory of Cell Proliferation and Differentiation of the Ministry of Education, School of Life Science, Peking University, Beijing I0087I, People's Republic of China; ${ }^{2}$ Department of Pathology, Zhongshan Hospital Xiamen University, Xiamen 36I004, People's Republic of China; ${ }^{3}$ Department of Rehabilitation Medicine, Peking University 3rd Hospital, Beijing I00191, People's Republic of China

*Both authors contributed equally to this work
Correspondence: Ji-Xiang Cao

Key Laboratory of Cell Proliferation and Differentiation of the Ministry of Education, School of Life Sciences, Peking University, Beijing 10087I, People's

Republic of China

Tel +86 I0 82802270

Email j_xcao@bjmu.edu.cn
Purpose: The cell division cycle 7 (CDC7) is a serine/threonine kinase that is essential for DNA replication in human cells which has been identified to play a critical role in multiple cancer types. However, the expression and clinical significance of CDC7 in ESCC has never been reported.

Patients and methods: CDC7 expression was detected in 30 ESCC and matched adjacent normal tissues, and a series of loss-of-function and gain-of-function assays were performed to evaluate the effects of $\mathrm{CDC} 7$ on the proliferation, migration and invasion, and chemoresistance of ESCC cells.

Results: The results showed that CDC7 was highly expressed in ESCC tissues compared with matched adjacent normal tissues. Functional studies demonstrated that knockdown of CDC7 inhibited proliferation by arresting ESCC cells in the G0/G1 phase and inducing apoptosis. Knockdown of CDC7 also inhibited cell migration and invasion in ESCC cells. Furthermore, knockdown of CDC7 sensitized ESCC cells to Cis and 5-FU.

Conclusion: Our results suggest that $\mathrm{CDC7}$ is highly expressed in ESCC tissues, and silencing CDC7 enhances chemosensitivity of ESCC cells, providing a new avenue for ESCC therapy.

Keywords: CDC7, ESCC, chemosensitivity, therapeutic target, proliferation, migration and invasion

\section{Introduction}

Esophageal cancer is one of the most aggressive and lethal malignancies, and the 5-year survival rate for patients with advanced esophageal cancer is still less than $25 \%{ }^{1,2}$ Esophageal cancer is generally categorized into two major histologic subtypes: EAC and ESCC. ESCC is the primary histological type of esophageal cancer in eastern Asia, particularly in People's Republic of China. ${ }^{3}$ Although several treatment modalities for ESCC have been developed, the prognosis in patients with ESCC remains poor and unsatisfactory. ${ }^{4-6}$ To improve the efficacy of ESCC treatment, combination therapies of preoperative chemotherapy or chemoradiotherapy followed by surgery have been developed. Chemotherapy-based combination regimens differ between patients but the prognosis is not satisfactory for nonresponders, probably because of chemotherapy resistance. ${ }^{7-9}$ Therefore, it is crucial to overcome resistance to improve prognosis for ESCC patients.

CDC7 is a conserved serine/threonine kinase that is of critical importance in the initiation of DNA replication and DNA damage stress. ${ }^{10,11}$ Overexpression of CDC7 has been reported in many human tumor cell lines and tissues, including ovarian cancer, ${ }^{12}$ colorectal cancer, ${ }^{13}$ lung cancers, ${ }^{14}$ malignant salivary gland tumors, ${ }^{15}$ and breast cancer, ${ }^{16}$ but has a very low or undetectable expression in normal tissues and 
cell lines. ${ }^{17}$ Accumulating evidence indicates that $\mathrm{CDC} 7$ silencing causes p53-independent apoptosis of tumor cells, but not normal cells. ${ }^{18,19}$ Furthermore, overexpression of CDC7 promotes tumor chemoresistance and survival via multiple pathways. ${ }^{20}$ Therefore, CDC7 becomes an attractive target for cancer therapy. ${ }^{21,22}$ However, the expression and the roles of $\mathrm{CDC} 7$ have never been reported in ESCC.

In this study, we analyzed the expression of $\mathrm{CDC} 7$ in esophageal cancer by using The Cancer Genome Atlas (TCGA) database and evaluated the expression of $\mathrm{CDC} 7$ in ESCC tissues and paired adjacent normal tissues by using IHC. Functionally, we found that downregulated CDC7 could improve the sensitivity of ESCC to chemotherapy.

\section{Materials and methods}

\section{Tissue specimens}

We obtained 30 primary ESCC tissues and paired adjacent normal tissues from the affiliated Zhongshan Hospital of Xiamen University during 2012-2016. All patients have given written informed consent and did not receive neoadjuvant/ adjuvant treatments before surgery. The pathological diagnosis of all specimens was confirmed by pathologist. This study was carried out in accordance with the principles of the Declaration of Helsinki and approved by the Research Ethics Committee of Xiamen University.

\section{Bioinformatics analysis}

TCGA (http://cancergenome.nih.gov/) provides researchers with comprehensive molecular characterization of multiple cancer types. CDC7 mRNA expression and clinical data from TCGA dataset for the esophagus cancer and normal samples were then analyzed on UALCAN (http://ualcan. path.uab.edu/), an easy to use, interactive web portal to perform in-depth analyses of TCGA gene expression data. ${ }^{23} \mathrm{In}$ addition, UALCAN also was used to analyze the association between CDC7 levels and clinical characteristics of esophagus cancer patients.

\section{Cell culture and treatments}

Human ESCC KYSE150 cells were purchased from the Cancer Hospital of the Chinese Academy of Medical Sciences (Beijing, People's Republic of China), and KYSE30 cells were obtained from the Cell Bank of the Chinese Academy of Sciences (Shanghai, People's Republic of China). Both Cells were maintained in RPMI 1640 medium supplemented with 10\% FBS (Hyclone, Thermo Fisher Scientific, Waltham, MA, USA) and grown at $37^{\circ} \mathrm{C}$ with $5 \% \mathrm{CO}_{2}$. Cis, 5-FU, and PHA-767491 were purchased from Sigma and were added to the cultures at the given time points.

\section{Small interfering RNA (siRNA) and transfection}

The siRNA sequences targeting CDC7 were synthesized by GenePharma (Shanghai, People's Republic of China), and the sequence was as follows: siCDC7, 5'-AAGCAGUCAAAGACUGUGGAU-3'; scrambled RNA, 5'-UUCUCCGAACGUGUCACGUTT-3'. Transfection was performed with Lipofectamine 2000 (Thermo Fisher Scientific) according to the manufacturer's instructions.

\section{RNA isolation and RT-qPCR}

Total RNA was isolated using Trizol reagent (Thermo Fisher Scientific). For mRNA detection, cDNA was prepared using RevertAid ${ }^{\text {TM }}$ First Strand cDNA Synthesis Kit (Thermo Fisher Scientific) according to the manufacturer's instructions. Gene expression was quantified by using the Power SYBR Green PCR Master Mix (Thermo Fisher Scientific). Primers were as follows: CDC7, 5'-AGTGCCTAACAGTGGCTGG-3' and 5'-CACGGTGAACAATACCAAACTGA-3'; GAPDH, 5'-TGTCAGTGGTGGACCTGACCT-3' and 5'-AGGGGAGATTCAGTGTGGTG-3'; and GAPDH was utilized as normalization.

\section{Western blotting}

For Western blotting analysis, cells were harvested and whole-cell extracts were prepared in lysis buffer. Protein concentration was determined using the BCA Protein Assay Reagent Kit (Thermo Fisher Scientific) according to the manufacturer's instructions. Protein samples were separated by $10 \%$ SDS-PAGE and transferred onto nitrocellulose membranes. The membranes were incubated in blocking solution consisting of $5 \% \mathrm{w} / \mathrm{v}$ non-fat milk in TBST at room temperature (RT) for 1 hour and imunoblotted with primary antibodies at $4^{\circ} \mathrm{C}$ overnight, followed by incubation with secondary antibody at RT for 1 hour. Protein bands were visualized using SuperSignal West Pico Chemiluminescent Substrate (Pierce, Rockford, IL, USA). Antibodies used for Western blotting assay were as follows: CDC7 (sc-56275, Santa Cruz Biotechnology Inc., Dallas, TX, USA); E-Cadherin (CST-14472, Cell Signaling Technology, Danvers, MA, USA); Snail (CST-3879, Cell Signaling Technology); Nrf2 (CST-12721, Cell Signaling Technology); Lamin B1 (CST-13435, Cell Signaling Technology); and $\alpha$-tubulin (PM054; MBL, Nagoya, Japan).

\section{Cell survival assay}

ESCC Cells were seeded in a 96-well plate and treated with the indicated molecules. Cell proliferation was determined using CellTiter 96 AQueous Non-Radioactive Cell 
Proliferation Assay (MTS) (Promega Corporation, Fitchburg, WI, USA) according to the manufacturer's instructions.

\section{Colony formation assay}

For the colony formation assay, ESCC cells were transfected or treated with the indicated molecules. ESCC cells were seeded into six-well plates at a density of $5 \times 10^{2}$ cells per well. After culture for 10 days, the colonies were fixed with $4 \%$ paraformaldehyde for 15 minutes and then stained with $0.1 \%$ crystal violet for 15 minutes. Visible cell colonies were imaged and counted.

\section{Wound-healing assay}

Cell migration was assayed using the wound-healing assay. ESCC cells were transfected or treated with the indicated molecules, ESCC cells were seeded into six-well plates and grown until they attained $80 \%-90 \%$ confluence. The layer of cells in each well was then scratched with a $10 \mu \mathrm{L}$ pipette tip and washed three times with PBS. The cells were maintained in fresh serum-free DMEM medium for 24 hours at $37^{\circ} \mathrm{C}$, $5 \% \mathrm{CO}_{2}$. The migration distance was quantified using the ImageJ software program.

\section{Invasion assay}

Cell invasion was assayed using the 24-well invasion chambers coated with $250 \mu \mathrm{g} / \mathrm{mL}$ Matrigel. ESCC cells were transfected or treated with the indicated molecules, and $1 \times 10^{4}$ cells in $200 \mu \mathrm{L}$ of medium without FBS were seeded in the upper chambers. A total of $500 \mu \mathrm{L}$ of culture medium (containing $10 \%$ FBS) were added to the lower chamber, and the cells were incubated for 24 hours. Next, the cells on the upper surface of the filters were removed, and the cells migrating through the filters were fixed with $4 \%$ paraformaldehyde and stained with $0.1 \%$ crystal violet. Cells that migrated through the filters were counted in five random fields using an inverted microscope. Each assay was repeated at least three times.

\section{IHC}

For IHC, slides were deparaffinized in xylene and rehydrated in a graded alcohol series. Antigen retrieval was performed in citrate buffer ( $\mathrm{pH}$ 6.0) via microwave treatment for 15 minutes. The slides were incubated with $3 \% \mathrm{H}_{2} \mathrm{O}_{2}$ for 10 minutes at RT to block endogenous peroxidase, followed by blocked with goat serum for 30 minutes at RT. The sections were incubated with a primary antibody against CDC7 (sc-56275, 1:100 dilution) at $4^{\circ} \mathrm{C}$ overnight. After incubated with HRP-conjugated secondary antibody for 30 minutes at $\mathrm{RT}$, the slides were stained with diaminobenzidine (DAB) and hematoxylin. The staining of $\mathrm{CDC} 7$ was analyzed by semi-quantitative method. The staining intensity (SI) was scored as follows: negative (0), weak (1), moderate (2), and strong (3). The percentage of positive cells (PP) was scored as follows: $\leq 5 \%(0) ; 6 \%-25 \%(1) ; 26 \%-50 \%(2) ; 51 \%-75 \%$ (3); and $\geq 75 \%$ (4). The IHS was calculated by the formula: $\mathrm{IHS}=\mathrm{SI} \times \mathrm{PP}$.

\section{Flow cytometry}

For the cell apoptosis assay, ESCC cells were transfected or treated with the indicated molecules and harvested for analysis 48 hours after transfection. Then apoptosis was evaluated using Annexin V/PI Apoptosis Detection Kit (Biosea, Beijing, People's Republic of China) according to manufacturer's instructions.

\section{Statistical analysis}

Student's $t$-test and Wilcoxon's rank-sum test were used for statistical analysis. All experiments were repeated in triplicates. All data are presented as the mean $\pm \mathrm{SD}$, and $P<0.05$ was considered statistically significant.

\section{Results}

\section{CDC7 is highly expressed in ESCC tissues}

To examine the expression level of $\mathrm{CDC} 7$ in esophageal carcinoma, we first analyzed data from TCGA, which includes 184 primary esophageal carcinoma samples and 11 normal esophageal tissues. Results from TCGA showed that the mRNA level of $\mathrm{CDC} 7$ was increased in esophageal carcinoma compared with normal tissue $(P=1.62 \mathrm{E}-12)$ (Figure 1A). To further assess the clinical relevance between CDC7 expression and the clinical characteristics of esophageal carcinoma patients, we observed that the expression of CDC7 was increased in all histology types (EAC and ESCC), all stages, and all tumor grades of esophageal carcinoma tissues compared with the normal tissue based on the data from UALCAN (Figure 1B-D).

Subsequently, we further verified the high expression of CDC7 in ESCC, we performed IHC staining to detect the expression of $\mathrm{CDC} 7$ in $30 \mathrm{ESCC}$ specimens and matched adjacent normal tissues. As shown in Figure 2, CDC7 protein level had positive expression in ESCC tissues, but had very low or undetectable expression in normal esophageal tissues (Figure 2A). Furthermore, quantitation data of IHC score revealed that $\mathrm{CDC} 7$ protein level was highly expressed in ESCC tissues $(P<0.0001)$ (Figure 2B). Taken together, our data indicate that $\mathrm{CDC} 7$ was highly expressed in ESCC tissues. 
A

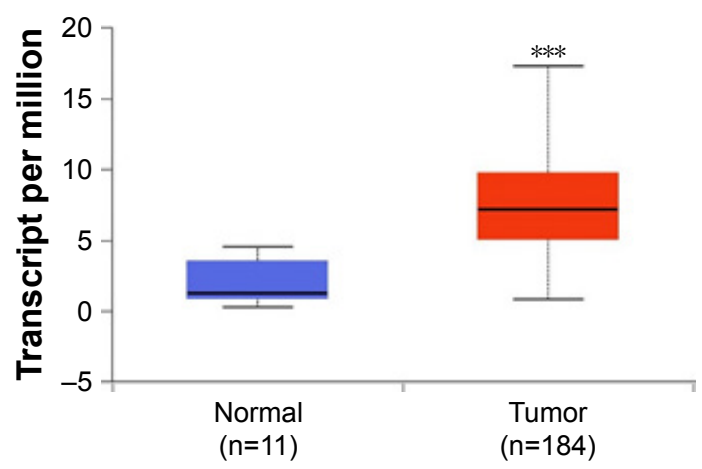

C

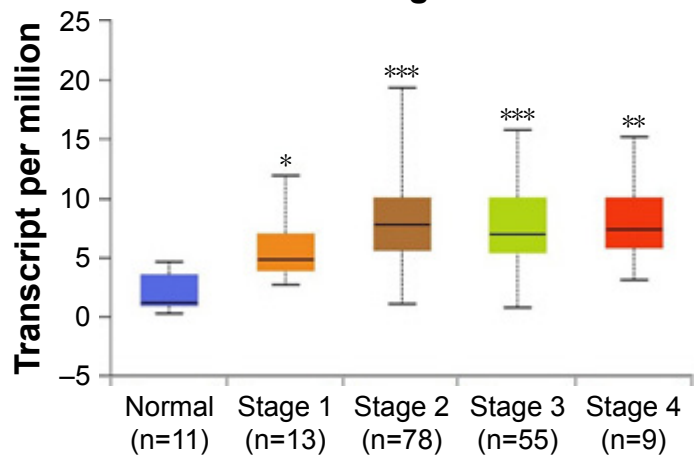

B

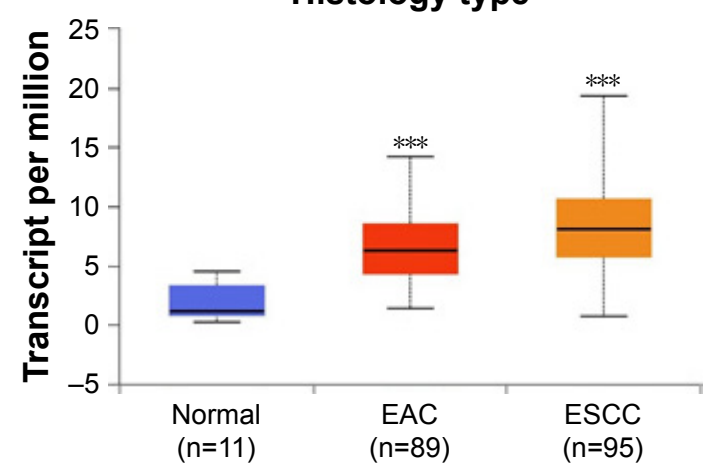

D

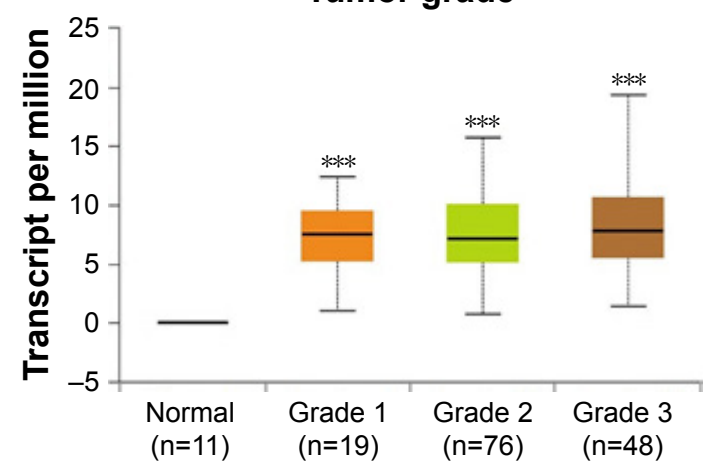

Figure I Box-whisker plots showing the expression of CDC7 in subgroups of esophageal carcinoma.

Notes: (A) Boxplot showing relative expression of CDC7 in normal and esophageal carcinoma tissues. (B and D) Boxplot showing relative expression of CDC7 in all histology types (B), all stages (C), and all tumor grades (D) of esophageal carcinoma vs normal tissues from TCGA dataset. $* P<0.05$; $* * P<0.0$ I; $* * * P<0.00$ I.

Abbreviations: EAC, esophageal adenocarcinoma; ESCC, esophageal squamous cell carcinoma; TCGA, The Cancer Genome Atlas.

\section{Effect of CDC7 on proliferation of ESCC cells}

To explore the function of CDC7 in ESCC, we first analyzed the expression of CDC7 in ESCC cell lines from CCLE database (https://portals.broadinstitute.org/ccle)
(Supplementary materials). KYSE150 cells and KYSE30 cells were selected for further analysis because the expression of CDC7 in KYSE150 cells was high and the expression of CDC7 in KYSE30 cells was low (Figure 3A). We silenced the expression of CDC7 in KYSE150 cells using CDC7
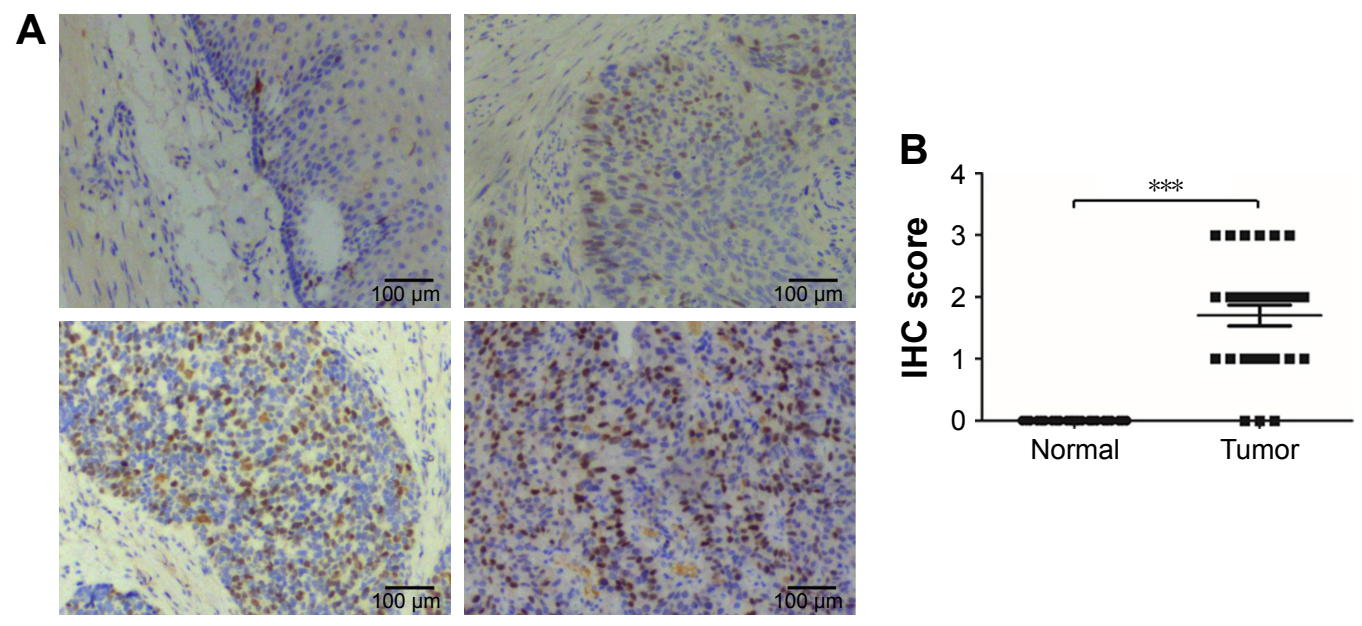

Figure 2 CDC7 is highly expressed in ESCC tissues.

Notes: (A) Representative IHC images of CDC7 expression in ESCC and matched adjacent normal tissues, top-left panel, negative staining in adjacent normal tissues; topright panel, weak staining in ESCC; bottom-left panel, and moderate staining in ESCC; bottom-right panel, strong staining in ESCC. Magnification: 100×. (B) The IHC score of CDC7 was determined in ESCC and matched adjacent normal tissues; ***P $<0.00$ I.

Abbreviations: ESCC, esophageal squamous cell carcinoma; IHC, immunohistochemistry. 
A

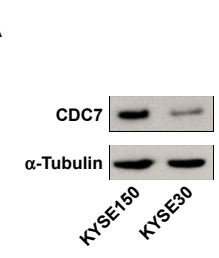

B

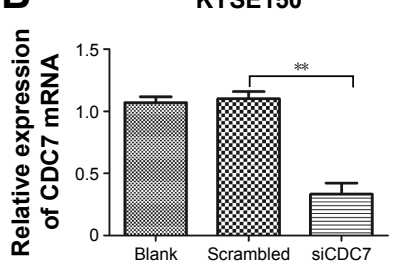

C

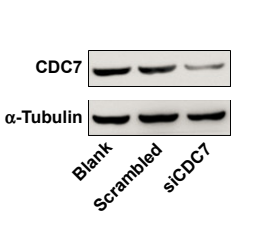

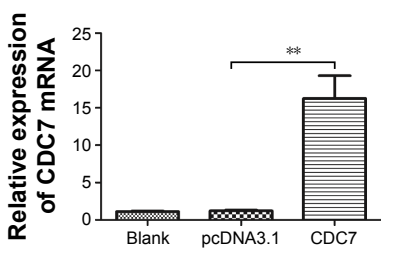

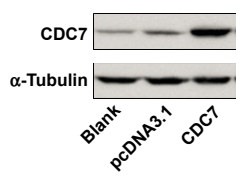

\section{D}

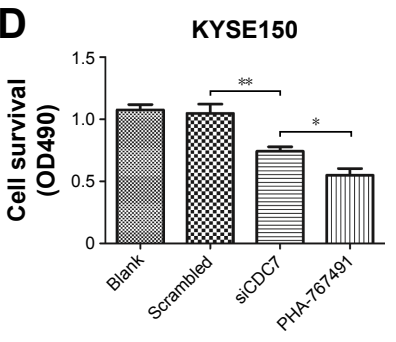

$\mathbf{F}$
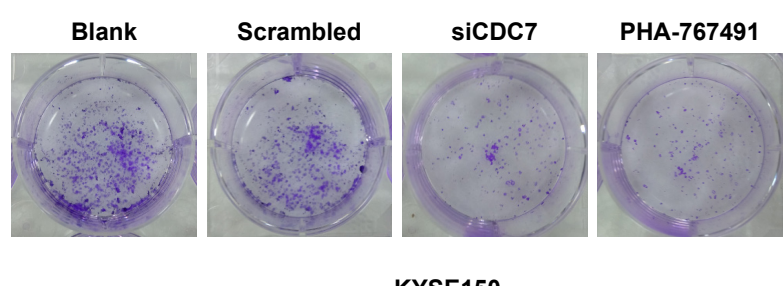

G

E

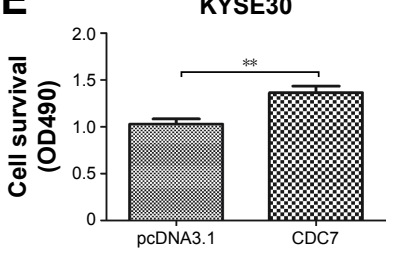

KYSE150

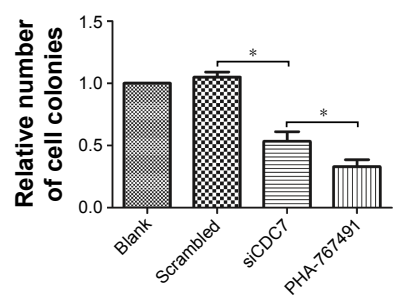

pcDNA3.1

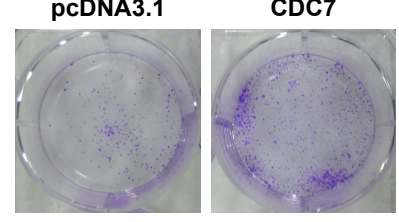

KYSE30

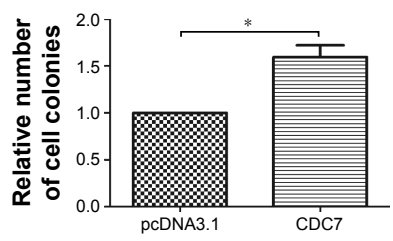

Figure 3 Effect of $\mathrm{CDC7}$ on proliferation of ESCC cells.

Notes: (A) Protein levels of CDC7 in ESCC cells were analyzed by Western blotting. (B and C) KYSEI 50 cells were transfected with siCDC7 for 48 hours and KYSE30 cells were transfected with pcDNA3.I-CDC7 for 48 hours. RT-qPCR and Western blotting were performed to examine CDC7 mRNA and protein levels, respectively. KYSEI 50 cells were transfected with siCDC7 or treated with PHA-76749I (2.5 $\mu$ M), and KYSE30 cells were transfected with pCDNA3.I-CDC7. (D and E) MTS assays were performed. (F and $\mathbf{G})$ Colony formation assays were performed. Data are presented as the mean $\pm \operatorname{SD}(n=3) ; * P<0.05 ; * * P<0.0$ I.

Abbreviations: ESCC, esophageal squamous cell carcinoma.

siRNA and overexpression of CDC7 in KYSE30 cells using pcDNA3.1-CDC7 vector. RT-qPCR and Western blotting were performed to confirm the efficiency of transfection (Figure $3 \mathrm{~B}$ and $\mathrm{C}, P=0.0019, P=0.0077$, respectively). Then, MTS assays were employed to evaluate the proliferation. Our results showed that downregulation of CDC7 in KYSE150 cells suppressed proliferation from 1.05 (scrambled siRNA) to 0.74 (Figure 3D, $P=0.0093$ ); in addition, inhibited CDC7 kinase activity through PHA-767491 in KYSE150 cells suppressed proliferation from 0.74 (siCDC7) to 0.55 (Figure 3D, $P=0.0248$ ), while overexpression of $\mathrm{CDC} 7$ in KYSE30 cells showed the opposite effects (Figure 3E, $P=0.0094$ ). Similar results were observed in colony formation assays (Figure $3 \mathrm{~F}$ and $\mathrm{G}, P=0.0384, P=0.0183$, and $P=0.0436$, respectively).

To further explore the reason of CDC7's function in cell proliferation, we assessed the cell cycle phase distribution by flow cytometry. Flow cytometry assays showed that downregulation of $\mathrm{CDC} 7$ by siRNA and inhibited $\mathrm{CDC} 7$ kinase activity through PHA-767491 in KYSE150 cells significantly increased the percentage of cells at the G0/G1 phase and decreased the percentage of cells at the $\mathrm{S}$ phase (Figure $4 \mathrm{~A}, P=0.0044, P=0.0002$, respectively). In contrast, overexpression of $\mathrm{CDC7}$ in KYSE30 cells significantly decreased the percentage of cells at the G0/G1 phase and increased the percentage of cells at the S phase (Figure 4B, $P=0.0067)$. We also investigated the effect of $\mathrm{CDC} 7$ on apoptosis in KYSE150 and KYSE30 cells. Downregulation of CDC7 in KYSE150 cells increased apoptosis from 6.9\% (scrambled siRNA) to $16.8 \%$ (Figure $4 \mathrm{C}, P=0.0178$ ); in addition, inhibited CDC7 kinase activity through PHA-767491 in KYSE150 cells increased apoptosis from 16.8\% (siCDC7) to $25.5 \%$ (Figure $4 \mathrm{C}, P=0.0488$ ), while we could not detect any apoptosis in CDC7-transfected KYSE30 cells compared with pcDNA3.1 transfection (Figure 4D). Taken together, our results suggest that the effect of $\mathrm{CDC} 7$ on proliferation of ESCC cells is by arresting the tumor cells at the G0/G1 phase and accelerating apoptosis.

\section{Effect of CDC7 on migration and invasion of ESCC cells}

To further investigate the effect of $\mathrm{CDC} 7$ on migration and invasion of ESCC cells, CDC7 was knocked down in KYSE150 cells using siRNAs and overexpressed in KYSE30 cells using pcDNA3.1-CDC7 vector. We also assess the effect of CDC7-kinase inhibitor PHA-767491 on migration 
A

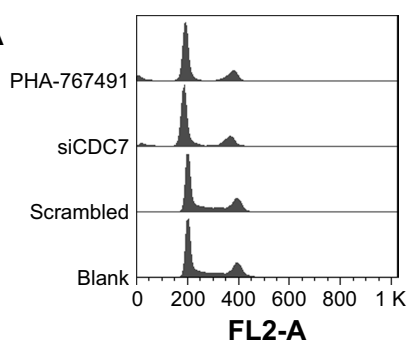

KYSE150
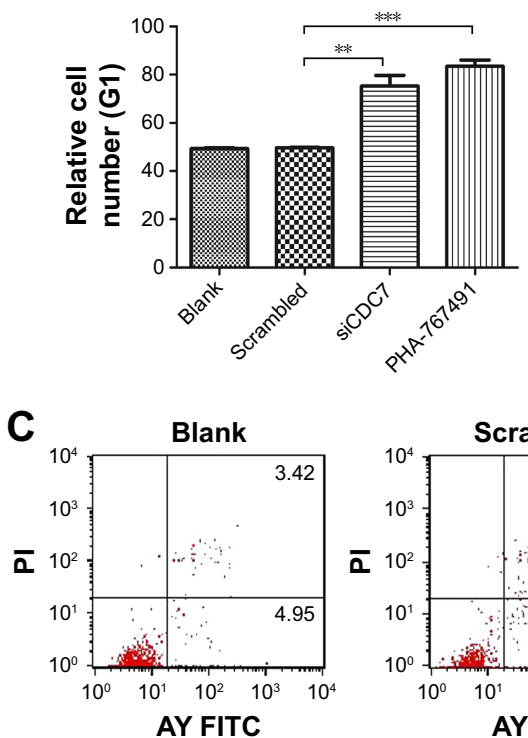

AY FITC

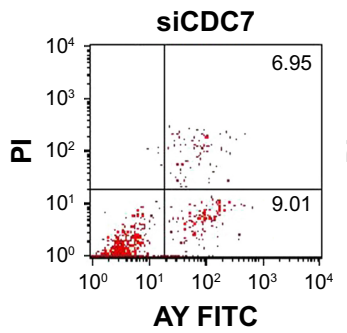

B

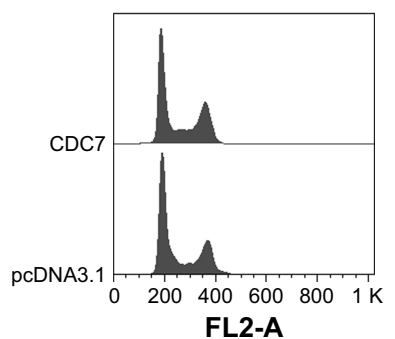

KYSE30

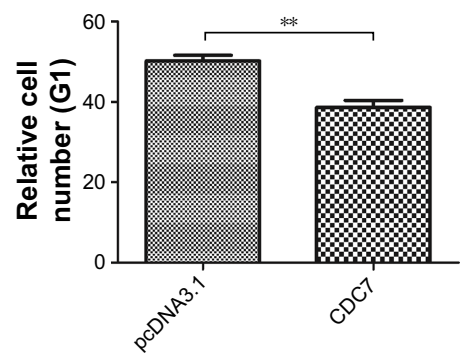

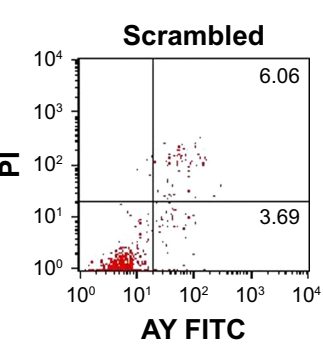
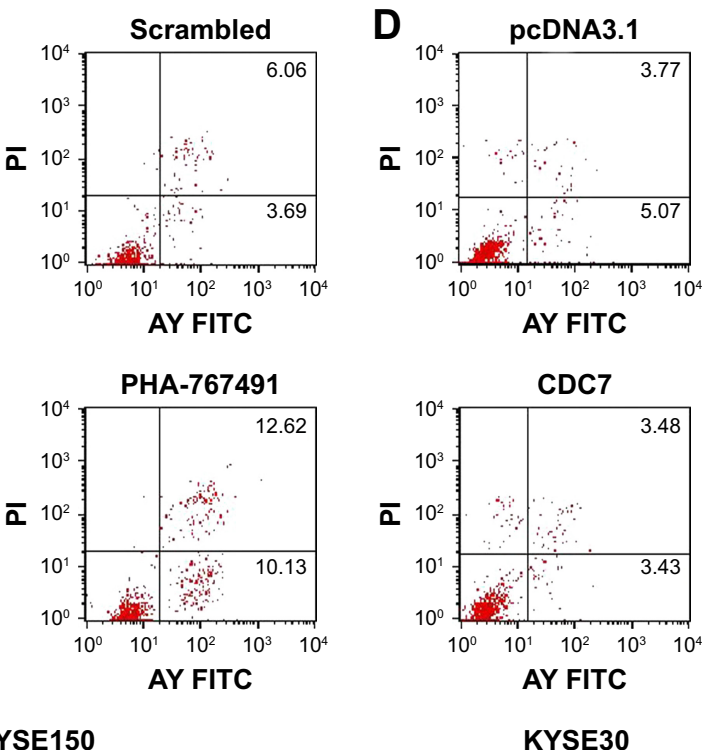

KYSE150
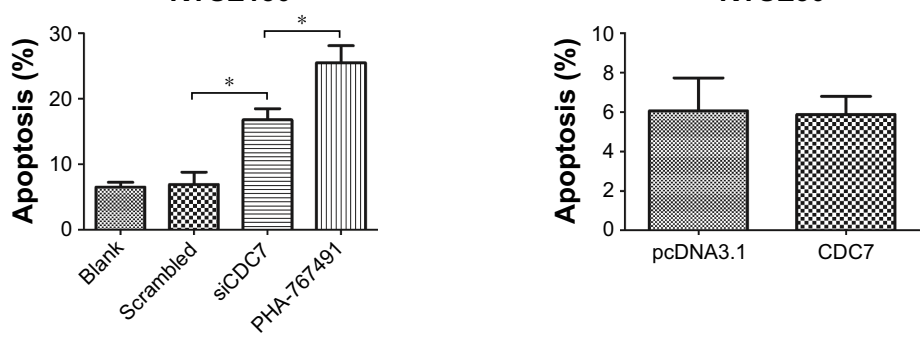

Figure 4 Effect of CDC7 on cell cycle transition and apoptosis of ESCC cells.

Notes: KYSEI 50 cells were transfected with siCDC7 or treated with PHA-76749I for 48 hours, and KYSE30 cells were transfected with pcDNA3.I-CDC7 for 48 hours. ( $\mathbf{A}$ and $\mathbf{B}$ ) Flow cytometry assays were performed to assess the effect of cell cycle transition. ( $\mathbf{C}$ and $\mathbf{D}$ ) Annexin V-FITC apoptosis assays were performed to assess the effect of apoptosis. Data are presented as the mean \pm SD $(n=3) ; * P<0.05 ; * * P<0.01 ; * * * P<0.001$.

Abbreviations: ESCC, esophageal squamous cell carcinoma; FITC, fluorescein isothiocyanate.

and invasion of ESCC cells. Our results showed that downregulation of $\mathrm{CDC7}$ suppressed the migration and invasion of KYSE150 cells compared with scrambled transfection (Figure 5A and $\mathrm{C}, P=0.0138, P=0.0350$, respectively) and overexpression of $\mathrm{CDC} 7$ promoted the migration and invasion of KYSE30 cells compared with pcDNA3.1 transfection (Figure 5B and D, $P=0.0079, P=0.0382$, respectively). In addition, similar results were observed in KYSE150 cells 

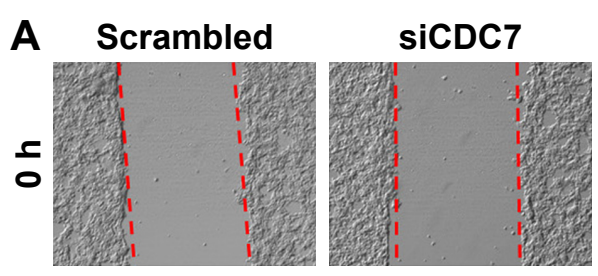

\section{PHA-767491}
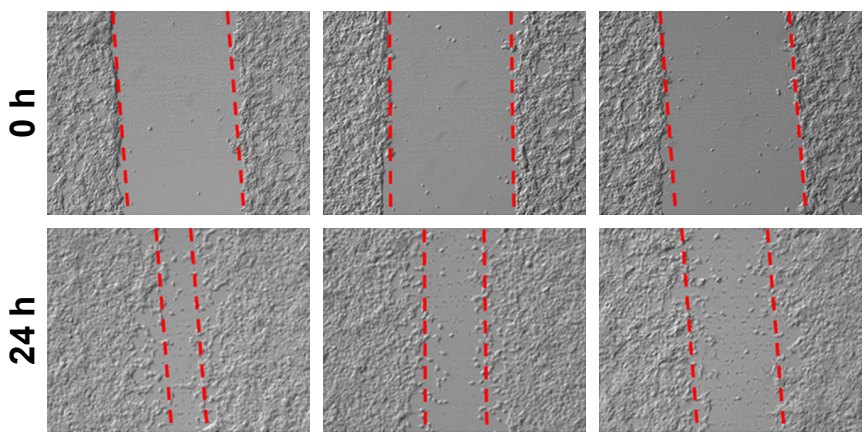

KYSE150
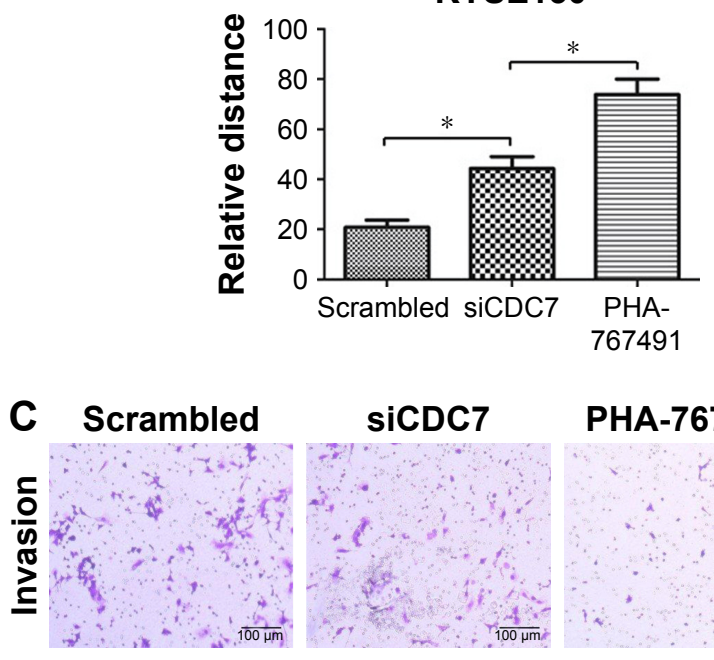

siCDC7

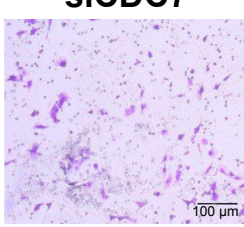

PHA-767491

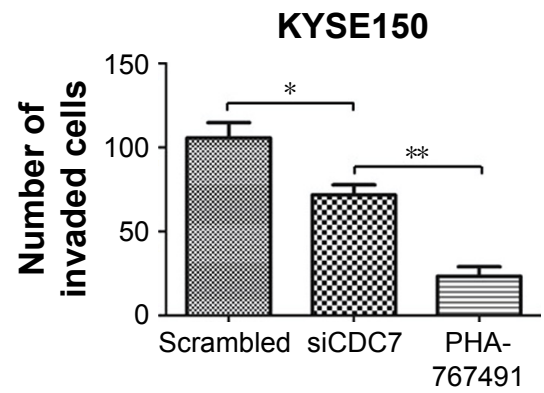

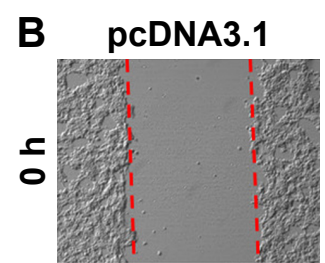
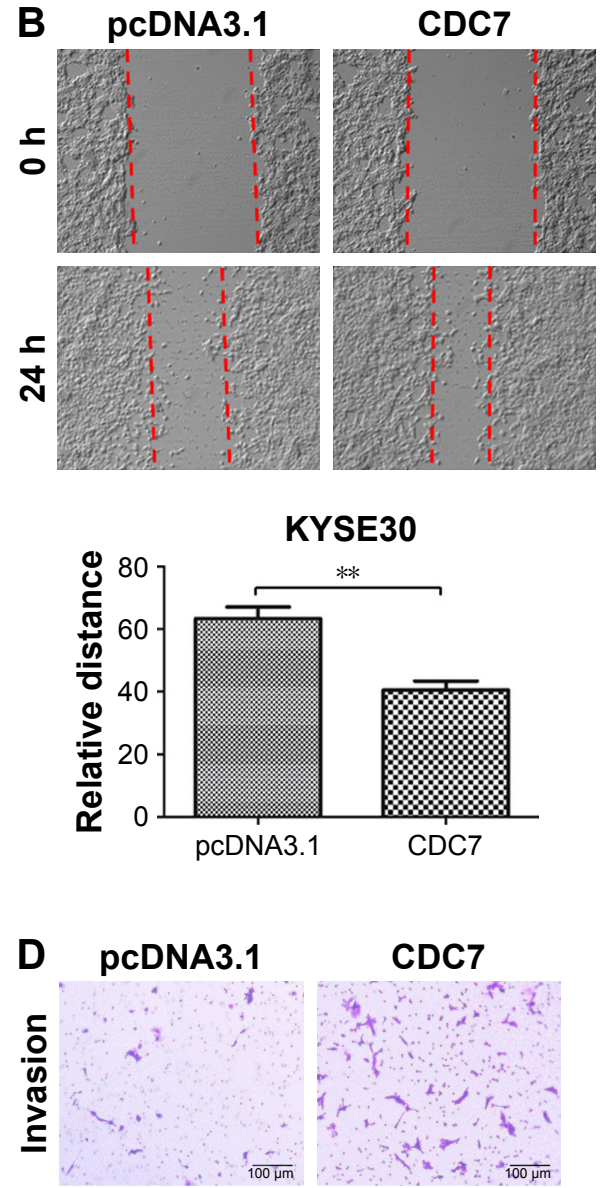

CDC7

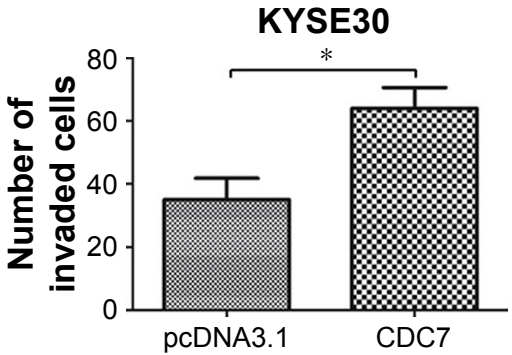

Figure 5 Effect of CDC7 on migration and invasion of ESCC cells.

Notes: KYSEI 50 cells were transfected with siCDC7 or treated with PHA-76749I for 48 hours, and KYSE30 cells were transfected with pcDNA3.I-CDC7 for 48 hours. ( $\mathbf{A}$ and $\mathbf{B}$ ) Effect of CDC7 on the migration was measured by wound-healing assays. The relative distances were calculated and compared in the bar graphs below. (C and $\mathbf{D}$ ) Effect of $\mathrm{CDC7}$ on the invasion was measured by transwell assays. The number of invaded cells were counted and compared in the bar graphs below. Magnification $\times 100$. Data are presented as the mean \pm SD $(n=3)(* P<0.05 ; * * P<0.0 I)$.

Abbreviations: ESCC, esophageal squamous cell carcinoma.

treated with PHA-767491 compared with siCDC7 transfection (Figure 5A and C, $P=0.0199, P=0.0042$, respectively). Taken together, these results indicate that $\mathrm{CDC} 7$ promotes tumor migration and invasion of ESCC cells.

\section{Effect of CDC7 on sensitivity of ESCC cells to chemotherapy}

To further determine the effect of $\mathrm{CDC} 7$ knockdown on the sensitivity of ESCC cells to chemotherapy, CDC7 expression was silenced by siRNA in KYSE150 cells and CDC7 expression was overexpressed using pcDNA3.1-CDC7 vector, then grown in the presence or absence of Cis or 5-FU, followed by testing cell survival and apoptosis. Results from MTS assay showed that Cis and 5-FU treatment resulted in significantly decreased cell survival in CDC7 knockdown cells as compared with their respective controls (Figure 6A), while overexpression of CDC7 in KYSE30 cells showed the opposite effects (Figure 6B). Furthermore, results from apoptosis 

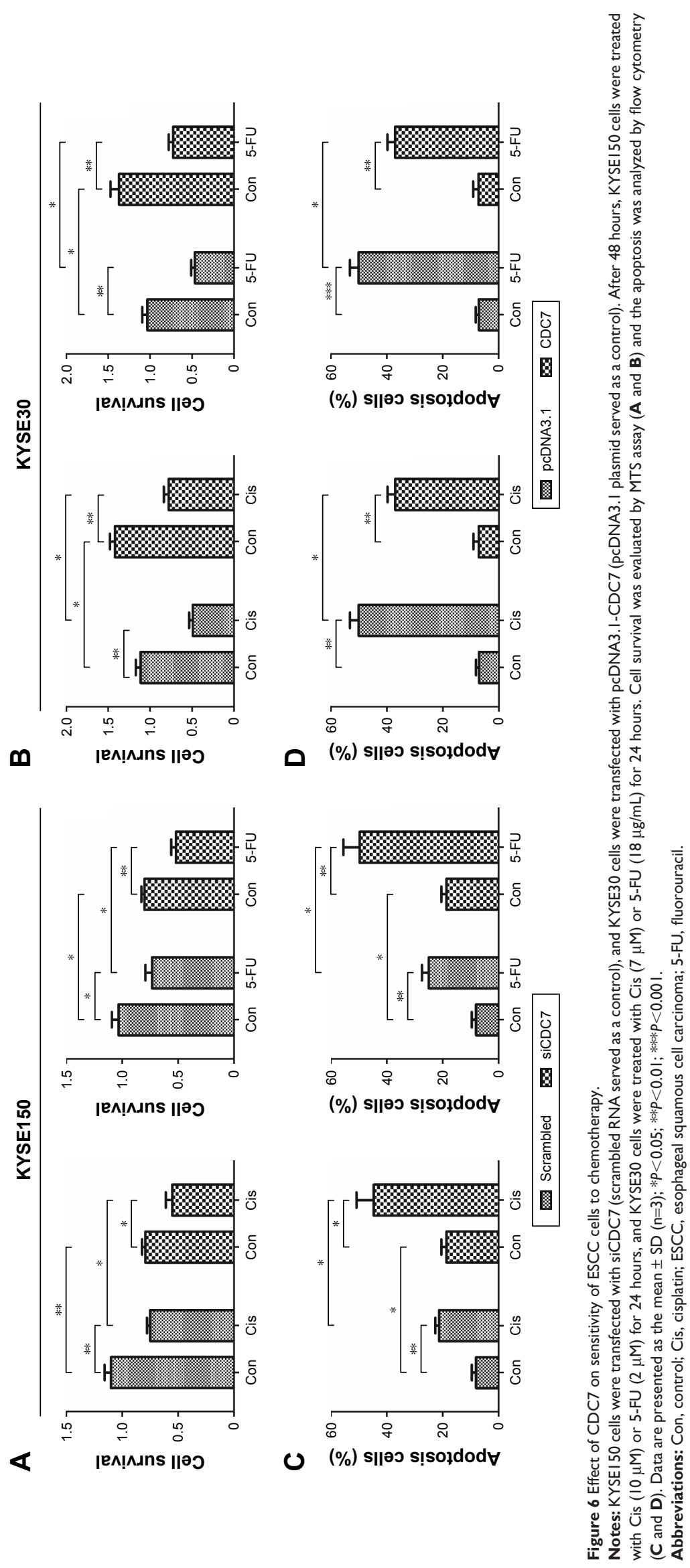
assay showed that the fraction of apoptotic cells were significantly increased in CDC7 knockdown cells as compared with their respective controls upon Cis and 5-FU treatment (Figure 6C), while overexpression of CDC7 in KYSE30 cells significantly reduced $\mathrm{Cis}$ and 5-FU-induced apoptotic cells (Figure 6D). These results suggest that knockdown of CDC7 sensitizes ESCC cells to chemotherapy.

\section{Discussion}

In the current study, we first evaluated the expression of CDC7 in ESCC tissues and matched adjacent normal tissues. In addition, we explored the relationship between $\mathrm{CDC} 7$ expression and the chemoresistance in human ESCC cells. Our data showed that CDC7 was highly expressed in ESCC tissues compared with matched adjacent normal tissues, and knockdown of CDC7 sensitizes ESCC cells to Cis and 5-FU. Therefore, our data suggest that high expression of CDC7 may be one of the reasons of chemoresistance in ESCC patients.

Systematic chemotherapy or neoadjuvant chemotherapy played an important role in the treatment of ESCC, but the efficacy of chemotherapy remains unsatisfactory. ${ }^{24,25}$ Chemoresistance was one of the major obstacles to achieve optimal chemotherapeutic outcomes. Therefore, it is urgent to uncover the potential mechanisms of chemoresistance to improve the survival and prognosis of patients with ESCC. Accumulating evidence have indicated that multiple molecules were involved in anticancer drug resistance of ESCC. ${ }^{9,26-31}$ For example, IL6 was found to promote chemoresistance in ESCC via CXCR 7, ${ }^{26}$ and downregulation of HOXA13 sensitizes human ESCC to chemotherapy and might regulate epithelial-to-mesenchymal transition (EMT). ${ }^{30}$ $\mathrm{CDC} 7$ has been reported to promote tumor chemoresistance and survival via multiple pathways. ${ }^{20}$ However, it is unclear whether $\mathrm{CDC7}$ is involved in chemoresistance in $\mathrm{ESCC}$ patients. To investigate whether $\mathrm{CDC} 7$ is involved in $\mathrm{ESCC}$ chemoresistance, we performed IHC to detect the expression of CDC7 in 30 ESCC tissues and matched adjacent normal tissues (Figure 2A and B). Our data showed that CDC7 was upregulated in ESCC tissues, suggesting that high expression of $\mathrm{CDC} 7$ may be one of the reasons of chemoresistance in ESCC patients. To further investigate the role of $\mathrm{CDC} 7$ in ESCC chemoresistance, we knocked down CDC7 in KYSE150 cells, MTS and flow cytometry were performed to evaluate Cis or 5-FU-induced inhibition and apoptosis in KYSE150 cells, respectively. MTS assay showed that knockdown of $\mathrm{CDC} 7$ decreased cell survival upon Cis or 5-FU exposure (Figure 6A). Meanwhile, we found that the knockdown of $\mathrm{CDC} 7$ promoted $\mathrm{Cis}$ or 5-FU-induced apoptosis in KYSE150 cells (Figure 6C), while overexpression of CDC7 in KYSE30 cells showed the opposite effects (Figure 6B and D). Consistent with Sasi et al's study, ${ }^{20}$ our data suggested that knockdown of $\mathrm{CDC} 7$ sensitizes ESCC cells to chemotherapy.

Silencing CDC7 in cancer cells inducing p53-independent apoptosis of tumor cells, but not normal cells, makes CDC7 a very promising target for drug development. Accumulating evidence has indicated that several specific small molecule inhibitors of the CDC7 kinase as a novel anticancer agent have been development, such as PHA767491, XL413, and 2,3-dihydrothieno[3,2-d]pyrimidin4(1H)-ones. ${ }^{32-35}$ To compare the activity between siCDC7 and CDC7-kinase inhibitor PHA-767491, we performed a series of experiments to assess the effect of proliferation, apoptosis, and migration and invasion after treatments. Our results showed that PHA-767491 suppressed proliferation, migration and invasion more significantly than siCDC7, and inducing more apoptosis. Liu et al reported that PHA767491 can function as an NRF2 inhibitor drug candidate for cancer therapy via redox modulation. ${ }^{36}$ In addition, Shen et al reported that blockage of Nrf2 suppresses the migration and invasion of ESCC cells in hypoxic microenvironment via EMT pathway. However, we did not observe the nuclear translocation or expression of Nrf2 regulated by siCDC7 in ESCC cells (Figure S1). Thus, these results suggested that PHA-767491-mediated effects on cancer cell proliferation, migration and invasion, and apoptosis may be due to its combined net inhibitory activity on both $\mathrm{Cdc} 7$ / CDK9 and NRF2 ${ }^{37}$ Furthermore, to determine the effect of CDC7 downregulation on EMT, we detected the expression of Snail and E-Cadherin by Western blotting; our results showed that CDC7 does not promote ESCC cells migration and invasion via EMT pathway (Figure S2). Using the UALCAN database, we have showed that CDC7 is significantly upregulated in ESCC tissues and the expression of CDC7 was increased in all stages, all tumor grade, and all histology type of esophageal carcinoma tissues compared with the normal tissue, suggesting that $\mathrm{CDC} 7$ may be an ideal broad-spectrum anticancer targets for esophageal carcinoma. Furthermore, we found that the depletion of CDC7 can inhibit cell proliferation and induce apoptosis in KYSE150 cells. Thus, CDC7 may be a therapeutic target which has multi-effect in ESCC therapy and may improve the outcome of ESCC patients. Currently, a phase I clinical study is ongoing at Cancer Research UK (NCT03096054) to assess the safety of LY3143921 hydrate (a CDC7 inhibitor) 
in adult patients with advanced solid tumors, including squamous carcinoma of the esophagus.

\section{Conclusion}

We have demonstrated that CDC7 was significantly upregulated in ESCC tissues. Depletion of CDC7 can inhibit proliferation, migration and invasion, and induce apoptosis of ESCC cells. Furthermore, downregulation of CDC7 partially ameliorates chemoresistance of ESCC cells. Overall, our data indicate that $\mathrm{CDC} 7$ can serve as a potential therapeutic target for treating ESCC patients.

\section{Abbreviations}

Cis, cisplatin; EAC, esophageal adenocarcinoma; ESCC, esophageal squamous cell carcinoma; 5-FU, fluorouracil; IHC, Immunohistochemistry; IHS, immunohistochemical score.

\section{Acknowledgments}

We thank Dr Peter Cherepanov (Imperial College London) for providing the pcDNA3.1-CDC7 constructs. This research was supported by the National Natural Science Foundation of China (Grant No 81502393).

\section{Disclosure}

The authors report no conflicts of interest in this work.

\section{References}

1. Jemal A, Bray F, Center MM, Ferlay J, Ward E, Forman D. Global cancer statistics. CA Cancer J Clin. 2011;61(2):69-90.

2. Pennathur A, Gibson MK, Jobe BA, Luketich JD. Oesophageal carcinoma. The Lancet. 2013;381(9864):400-412.

3. Chen W, Zheng R, Zhang S, et al. Cancer incidence and mortality in China, 2013. Cancer Lett. 2017;401:63-71.

4. Tachimori Y, Ozawa S, Numasaki H, et al; Registration Committee for Esophageal Cancer of the Japan Esophageal Society. Comprehensive registry of esophageal cancer in Japan, 2010. Esophagus. 2017;14(3):189-214.

5. Tachimori Y, Ozawa S, Numasaki H, et al; Registration Committee for Esophageal Cancer of the Japan Esophageal Society. Comprehensive registry of esophageal cancer in Japan, 2011. Esophagus. 2018;15(3):127-152.

6. Tachimori Y, Ozawa S, Numasaki H, et al; Registration Committee for Esophageal Cancer of the Japan Esophageal Society. Comprehensive registry of esophageal cancer in Japan, 2009. Esophagus. 2016;13:110-137.

7. Miyata H, Yoshioka A, Yamasaki M, et al. Tumor budding in tumor invasive front predicts prognosis and survival of patients with esophageal squamous cell carcinomas receiving neoadjuvant chemotherapy. Cancer. 2009;115(14):3324-3334.

8. Tanaka K, Miyata H, Sugimura K, et al. miR-27 is associated with chemoresistance in esophageal cancer through transformation of normal fibroblasts to cancer-associated fibroblasts. Carcinogenesis. 2015; 36(8):894-903.

9. Shi Q, Shen LY, Dong B, et al. The identification of the ATR inhibitor VE-822 as a therapeutic strategy for enhancing cisplatin chemosensitivity in esophageal squamous cell carcinoma. Cancer Lett. 2018; 432:56-68.
10. Sclafani RA, Holzen TM. Cell cycle regulation of DNA replication. Annu Rev Genet. 2007;41:237-280.

11. Labib K. How do Cdc7 and cyclin-dependent kinases trigger the initiation of chromosome replication in eukaryotic cells? Genes Dev. 2010; 24(12):1208-1219.

12. Kulkarni AA, Kingsbury SR, Tudzarova S, et al. Cdc7 kinase is a predictor of survival and a novel therapeutic target in epithelial ovarian carcinoma. Clin Cancer Res. 2009;15(7):2417-2425.

13. Melling N, Muth J, Simon R, et al. Cdc7 overexpression is an independent prognostic marker and a potential therapeutic target in colorectal cancer. Diagn Pathol. 2015;10:125.

14. Datta A, Ghatak D, Das S, et al. p53 gain-of-function mutations increase Cdc7-dependent replication initiation. EMBO Rep. 2017;18(11):2030-2050.

15. Jaafari-Ashkavandi Z, Ashraf MJ, Abbaspoorfard AA. Overexpression of $\mathrm{CDC} 7$ in malignant salivary gland tumors correlates with tumor differentiation. Braz J Otorhinolaryngol. 2017; pii: S1808-8694(17)30211-2.

16. Choschzick M, Lebeau A, Marx AH, et al. Overexpression of cell division cycle 7 homolog is associated with gene amplification frequency in breast cancer. Hum Pathol. 2010;41(3):358-365.

17. Bonte D, Lindvall C, Liu H, Dykema K, Furge K, Weinreich M. Cdc7Dbf4 kinase overexpression in multiple cancers and tumor cell lines is correlated with p53 inactivation. Neoplasia. 2008;10(9):920-924.

18. Montagnoli A, Tenca P, Sola F, et al. Cdc7 inhibition reveals a p53dependent replication checkpoint that is defective in cancer cells. Cancer Res. 2004;64(19):7110-7116.

19. Im JS, Lee JK. ATR-dependent activation of $\mathrm{p} 38$ MAP kinase is responsible for apoptotic cell death in cells depleted of Cdc7. J Biol Chem. 2008;283(37):25171-25177.

20. Sasi NK, Bhutkar A, Lanning NJ, MacKeigan JP, Weinreich M. DDK Promotes tumor chemoresistance and survival via multiple pathways. Neoplasia. 2017;19(5):439-450.

21. Swords R, Mahalingam D, O’Dwyer M, et al. Cdc7 kinase - a new target for drug development. Eur J Cancer. 2010;46(1):33-40.

22. Montagnoli A, Moll J, Colotta F. Targeting cell division cycle 7 kinase: a new approach for cancer therapy. Clin Cancer Res. 2010;16(18): 4503-4508.

23. Chandrashekar DS, Bashel B, Balasubramanya SAH, et al. UALCAN: a portal for facilitating tumor subgroup gene expression and survival analyses. Neoplasia. 2017;19(8):649-658.

24. Smyth EC, Lagergren J, Fitzgerald RC, et al. Oesophageal cancer. Nat Rev Dis Primers. 2017;3:17048.

25. Nakajima M, Kato H. Treatment options for esophageal squamous cell carcinoma. Expert Opin Pharmacother. 2013;14(10):1345-1354.

26. Qiao Y, Zhang C, Li A, et al. IL6 derived from cancer-associated fibroblasts promotes chemoresistance via CXCR7 in esophageal squamous cell carcinoma. Oncogene. 2018;37(7):873-883.

27. Liu ZC, Cao K, Xiao ZH, et al. VRK1 promotes cisplatin resistance by up-regulating c-MYC via c-Jun activation and serves as a therapeutic target in esophageal squamous cell carcinoma. Oncotarget. 2017;8(39): 65642-65658.

28. Zhang Z, Wen P, Li F, et al. HIPK2 inhibits cell metastasis and improves chemosensitivity in esophageal squamous cell carcinoma. Exp Ther Med. 2018;15(1):1113-1118.

29. Liu B, Wang C, Chen P, Cheng B, Cheng Y. RACKI induces chemotherapy resistance in esophageal carcinoma by upregulating the PI3K/AKT pathway and Bcl-2 expression. Onco Targets Ther. 2018;11:211-220.

30. Shi Q, Shen L, Dong B, et al. Downregulation of HOXA13 sensitizes human esophageal squamous cell carcinoma to chemotherapy. Thorac Cancer. 2018;9(7):836-846.

31. Sun Y, Zhai L, Ma S, et al. Down-regulation of RIP3 potentiates cisplatin chemoresistance by triggering HSP90-ERK pathway mediated DNA repair in esophageal squamous cell carcinoma. Cancer Lett. 2018;418:97-108.

32. Montagnoli A, Valsasina B, Croci V, et al. A Cdc7 kinase inhibitor restricts initiation of DNA replication and has antitumor activity. Nat Chem Biol. 2008;4(6):357-365. 
33. Koltun ES, Tsuhako AL, Brown DS, et al. Discovery of XL413, a potent and selective CDC7 inhibitor. Bioorg Med Chem Lett. 2012;22(11): 3727-3731.

34. Kurasawa O, Oguro Y, Miyazaki T, et al. Identification of a new class of potent $\mathrm{Cdc} 7$ inhibitors designed by putative pharmacophore model: synthesis and biological evaluation of 2,3-dihydrothieno[3,2-d] pyrimidin-4(1H)-ones. Bioorg Med Chem. 2017;25(7):2133-2147.

35. Kurasawa O, Homma M, Oguro Y, et al. 2-Aminomethylthieno[3,2-d] pyrimidin-4(3H)-ones bearing 3-methylpyrazole hinge binding moiety: highly potent, selective, and time-dependent inhibitors of $\mathrm{Cdc} 7 \mathrm{kinase}$. Bioorg Med Chem. 2017;25(14):3658-3670.
36. Liu HY, Tuckett AZ, Fennell M, Garippa R, Zakrzewski JL. Repurposing of the CDK inhibitor PHA-767491 as a NRF2 inhibitor drug candidate for cancer therapy via redox modulation. Invest New Drugs. 2018; 36(4):590-600.

37. Shen H, Yang Y, Xia S, Rao B, Zhang J, Wang J. Blockage of Nrf2 suppresses the migration and invasion of esophageal squamous cell carcinoma cells in hypoxic microenvironment. Dis Esophagus. 2014; 27(7):685-692. 


\section{Supplementary materials}

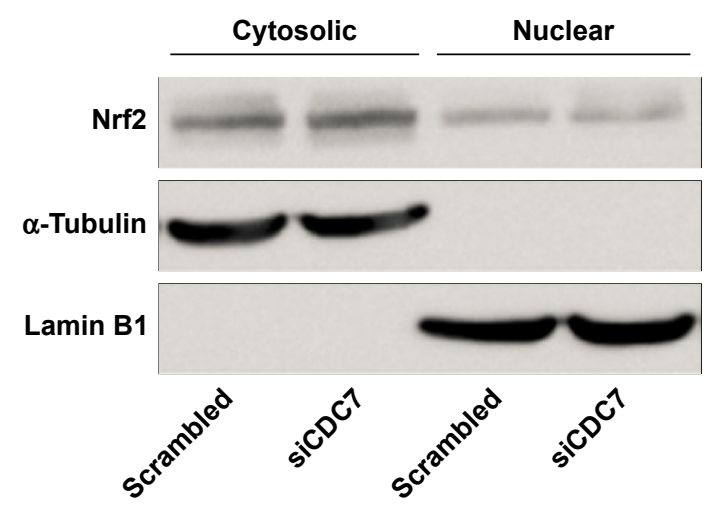

Figure SI Expression of Nrf2 in cytosolic and nuclear of KYSEI50 cells after transfected with siCDC7.

A

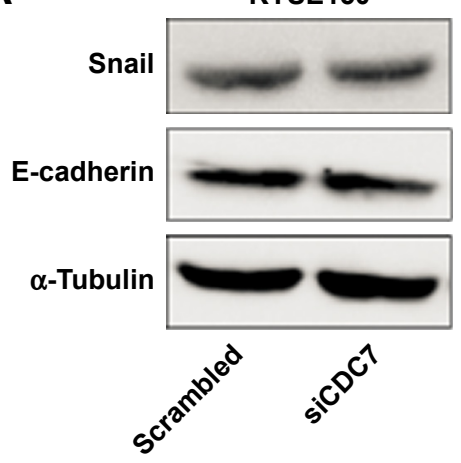

B

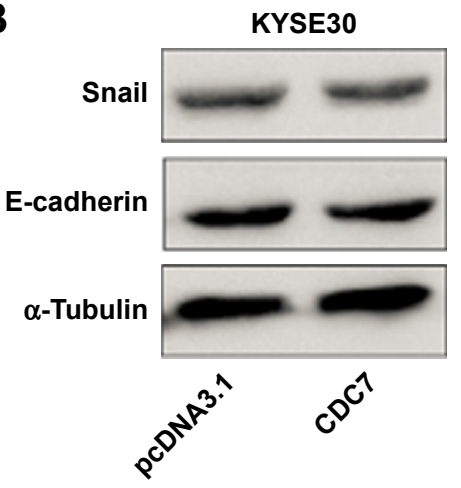

Figure S2 Expression of Snail and E-cadherin in ESCC cells after transfected with siCDC7 or pcDNA3.I-CDC7.

Notes: (A) KYSEI50 cells were transfected with siCDC7 for 48 hours, Western blotting were performed to assess the effect of EMT. (B) KYSE30 cells were transfected with pcDNA3.I-CDC7 for 48 hours, Western blotting were performed to assess the effect of EMT.

Abbreviations: EMT, epithelial-to-mesenchymal transition; ESCC, esophageal squamous cell carcinoma.

\section{Publish your work in this journal}

OncoTargets and Therapy is an international, peer-reviewed, open access journal focusing on the pathological basis of all cancers, potential targets for therapy and treatment protocols employed to improve the management of cancer patients. The journal also focuses on the impact of management programs and new therapeutic agents and protocols on patient perspectives such as quality of life, adherence and satisfaction. The manuscript management system is completely online and includes a very quick and fair peer-review system, which is all easy to use. Visit http://www.dovepress.com/testimonials.php to read real quotes from published authors. 